\title{
CLINICAL EFFICACY OF GIOMER VERSUS SODIUM FLUORIDE VARNISH FOR MANAGEMENT OF HYPERSENSITIVITY: RANDOMIZED CONTROL TRAIL
}

\author{
Nourane Y. Ali", Olfat Hassanein ${ }^{* *}$, Heba S. Hamza ${ }^{* * *}$ and Maha El Baz ${ }^{* * * *}$
}

\begin{abstract}
Aim: to assess the clinical effectiveness of Giomer varnish in comparison to sodium fluoride in reducing dentin hypersensitivity.

Methodology: Using a Split mouth design, 66 participants (132 teeth) of the outpatient clinic in the Faculty of dentistry, Cairo University, were enrolled. Each participant should have at least two sensitive teeth in different sides of the same jaw, with a visual analogue scale score two or more. Teeth were examined by using different stimuli: Evaporative, thermal and tactile stimuli. Sides were categorized randomly into two groups according to the desensitizing agents used either sodium fluoride or Giomer varnish. Degree of Hypersensitivity was assessed by visual analogue scale (VAS) before the application of the agents $\mathrm{T}_{1}$, immediately after $\mathrm{T}_{2}, 1$ week $\mathrm{T}_{3}$ and one month later $\mathrm{T}_{4}$. Data were analyzed using Two way ANOVA, partial eta-squared, Kruskal-Wallis, Bonferronicorrected Wilcoxon, Mann-Whitney test and Chi-square tests. Absolute risk, relative risk, relative risk reduction with $95 \%$ confidence interval of dentin hypersensitivity were calculated.
\end{abstract}

Results: Sodium fluoride exhibited high VAS scale at $\mathrm{T}_{1} 3.39(1.11), \mathrm{T}_{2} 3.21(0.99)$ and $\mathrm{T}_{4}$ 3.13(1.01) with a non-statistical difference between them. While at $\mathrm{T}_{3}$ statistically lower VAS scale $1.30(0.9)$ was shown in this group. Giomer exhibited high VAS scale at $\mathrm{T}_{1} 3.79(1.5)$ followed by $\mathrm{T}_{2} 2.17(0.57)$ and $\mathrm{T}_{4} 2.56(1.87)$ with a non-statistical difference between them. While at $\mathrm{T}_{3}$ lower VAS scale $0.17(0.57)$ was shown in this group. Influence of different assessment times within each agent was statistically significant at $\mathrm{P}<0.0001$. At $\mathrm{T} 2$ and $\mathrm{T} 3$, Giomer revealed statistically lower VAS scale and it exhibited a relative risk reduction by $83 \%$ at $\mathrm{T}_{3}$ and $24 \%$ at $\mathrm{T}_{4}$.

Conclusion: Giomer varnish is a promising desensitizing agent, which can replace sodium fluoride varnish in management of dentin hypersensitivity.

* Assistant Professor, Conservative Dentistry Department, Faculty of Dentistry, Egyptian Russian University, MSc Degree Candidate, Conservative Dentistry Department, Faculty of Dentistry, Cairo university.

** Professor and Head of Conservative Dentistry Department in Cairo University.

*** Professor of Conservative Dentistry Department in Cairo University.

**** Associate Professor of Conservative Dentistry Department in Cairo University. 


\section{INTRODUCTION}

Dentin hypersensitivity is a drastic problem, which threaten many dental patients. According to the last published systematic review and meta analysis at 2019, the prevalence of dentin hypersensitivity is being $33.5 \%$ among the population. Moreover, it was determined that young adults with age range from 18 to 44 years exhibited high percentage (43.9\%) of such problem (Zeola et al. 2019). Individuals with enamel loss due to any reason(s), gingival recession or those who undergo tooth whitening are more susceptible to have hypersensitive dentin. It is also stated that women are at a higher risk (Cunha-Cruz et al. 2013).

As a consequence of enamel loss, dentin becomes sensitive due to its exposure to different stimuli like chemical, thermal, tactile or osmotic (Orchardson and Collins 1987). The characteristics features of dentin hypersensitivity are being short and sharp pain (Bartold 2006). There are many theories that explain the pain mechanism and the most accepted one is the hydrodynamic theory (Brännström and Aström 1972).

Several teeth in one area of the mouth or only one specific tooth can be affected (Que et al. 2010, Amarasena et al. 2011). Careful diagnosis of such condition necessitates precise differentiation of its signs and symptoms from the other causes of tooth sensitivity, which may be resulted from dental caries, micro leakage, cracked tooth or fractured restoration (Addy 2002). With the innovation and the development of the sciences, the concept of the prevention becomes more important than curing.

Therefore, dentists dealing with dentin hypersensitivity must shift from the invasive restorative modalities to non-invasive techniques. Such techniques either reduce the discomfort of hypersensitivity by blocking the exposed dentinal tubules with occluding materials or use some depolarizing agents that reducing the excitability of the intra dental nerves (Orchardson and Gillam 2000).
Management of dentin hypersensitivity can be achieved by using self-performed therapy at home or specially applied agents in the dental office. There are many known desensitizing agents, which can reduce the hypersensitivity like Stronium chloride, sodium fluoride or potassium oxalate. Each one reduces the dentinal hypersensitivity by its own way and each one has different long lasting effects. The dentist must identify and select the best desensitizing agent to be used in the treatment. Sodium fluoride varnish is considered the gold standard desensitizing agent however, some drawbacks appeared with its use. Fluoride based agents appeared in 90's but it was clarified that they had a transient effect and their high concentration irritated the odontoblasts and became highly toxic (Vieria and Santiago 2009).

The Giomer varnish containing surface prereactive glass particles (S-PRG) is a light-cured protective varnish and it was introduced to promote prolonged protection for the hypersensitive dentin (Samuel et al. 2015). However, it has only one clinical trial which tested its clinical efficacy and so there is no clear evidence about its effectiveness (Ravishankar et al. 2018). The null hypothesis tested in this thesis is that there is no difference between the clinical effectiveness of Giomer in comparison to Sodium Fluoride varnish in reducing dentin hypersensitivity.

\section{MATERAILS AND METHODS}

\section{The following materials were used in this study}

\section{BiFluorid 10}

It is a varnish, which has $5 \%$ sodium fluoride varnish (equal to 22.600-ppm fluoride) and 5\% calcium fluoride (Voco, Germany). It is used for professional application as a treatment for dentin hypersensitivity. The sodium fluoride is considered the most commonly used desensitizing agent in treatment of dentin hypersensitivity, therefore it was selected to be the comparator in this study. 
The BiFluorid 10 is one of the compatible desensitizing agents in the treatment of dentin hypersensitivity because it is composed of natural ingredients and absence of methacrylate and glutaraldehyde.(Ravishankar et al. 2018).

\section{PRG Barrier coat}

Such coat is a Giomer varnish from Shofu Dental Corp., San Marcos, CA, USA). It is a light cured Surface-partially reacted glass (S-PRG) filler particles with a multifunctional glass core embedded in a resin matrix. It has an immediate and long lasting effect. The (S-PRG) favors the release of fluoride ions and its recharging that aid in remineralization and protection of the tooth structure in a way similar to the glass ionomer. The fluoride ions aid in the neutralization of the acidity of the oral cavity and decrease the plaque accumulation. (Halabo 2015).

The trial protocol was designed following the SPIRIT 2013 statement (Chan et al.2013) and approved from Evidence Based Dentistry Committee, Faculty of Dentistry Cairo University. The protocol and the template of informed consent form were reviewed and approved by the Ethics Committee of scientific research, Faculty of Dentistry - Cairo University in December 2018 (Approval No. 181221). The trial was registered in Protocol registration and results system (ClinicalTrials.gov PRS) with an identification number NCT03818945.

Regarding the design, it is a single Centered, triple blinded (participant, assessor and the data analyzer were blinded); two parallel armed randomized control clinical trial. The study was carried out on 66 adult Participants attending to the operative clinic in The Faculty of Dentistry, Cairo University, Egypt as they complained and were clinically diagnosed of dentin hypersensitivity. The researcher had the ultimate responsibility for all activities associated with the conduction of a research project including recruitment of Participants, explaining and performing the procedures to them.
Eligibility criteria of participants: (Athuluru et al.2017)

\section{Inclusion criteria of participants:}

- No gender restriction (Males or Females).

- Age: $18-40$ years old.

- Good oral hygiene.

- Co-operative who show interest to participate in the study and welling to sign the informed consent.

\section{Exclusion criteria of participants:}

- Allergic to the ingredients used in the study.

- Gastrointestinal disorders. (They have repeated vomiting attacks).

- Medically compromised. (They cannot tolerate the procedures).

- Taking desensitizing treatment in the last 6 months. (It affects the outcome)

- Taking anti-inflammatory drugs. (It affects the hypersensitivity perception)

- Pregnant females. ( They complain from nausea and repeated vomiting attacks)

- Smokers. (Smoking will affect the oral hygiene and periodontal health)

\section{Clinical procedures:}

Each tooth was tested using different stimuli as mentioned below after retracting the soft tissue by placing check retractor;

Evaporative stimulus was conducted by using a conventional dental unit air syringe, which was placed approximately $5 \mathrm{~mm}$ away from the tooth surface. The air temperature was $25^{\circ} \mathrm{C} \pm 3^{\circ} \mathrm{C}$ at pressure $0.5 \mathrm{~N} / \mathrm{mm}^{2}$. ( Hall et al. 2017) The duration of air blast ranged from 1-5 seconds according to participants response. As soon as the participants reported pain, the stimulus was stopped, and pain intensity was recorded. 
Thermal stimulus was assessed by using anaesthetic carpule kept in the refrigerator at $4^{\circ} \mathrm{C}$ for at least 24 hours till it became ice then it is removed from refrigerator immediately before testing. The carpule was placed on the tooth for one to five seconds according to participants' response as mentioned in evaporative testing.

Tactile stimulus by using the tip of sharp explorer No.3 (Hu-Friedy, Chicago, IL) which gently scratch the entire exposed tooth surface in apico-coronal direction with short strokes. The force applied with the stroke was standardized as the examination was done by single operator for all participants.

The participants pointed out the degree of sensitivity on the VAS scale (0-10) chart.Participants who had degree of dentin hypersensitivity two or more were enrolled. The scale was shown in a Plastic card with figures of facial expressions expressing degree of pain, color coded, and numbers guided for easy figuring out the degree of pain. There was a minimum of 5 min delay between the end of each assessment of hypersensitivity and the beginning of the next assessment to allow the tooth to recover. For each participants the highest respond from the all used stimuli, either evaporative, thermal or tactile, was recorded as the hypersensitivity scale. Case report form was specially designed for this study to be used in recording the outcome for each participant.

Way of assessment regarding the stimuli used and scoring system for dentin hypersensitivity was conducted according to a systematic review by (Gernhardt in 2013). It was revealed that the tactile, thermal and evaporative stimuli are the most commonly and recommended stimuli used in the evaluation of dentin hypersensitivity because they are more controllable. Participants' response to the degree of hypersensitivity was tested in the literature by using either verbal rating scale or visual analogue scale. The verbal rating scale are used to evaluate the level of pain by using several pain descriptors like no pain, weak, mild, moderate, strong, intense and agonizing. However, the main disadvantage is it cannot offer enough descriptions for case. While the VAS scale, it can be graded by using a line of $10 \mathrm{~cm}$ length with two extremities representing the absolute minimum and maximum pain level or validated graphic pain scale such as faces pain scale or combination between both.

\section{Application of Giomer Varnish (PRG Barrier coat)}

After opening the sealed envelope and knowing the code of Participants, while the check retractor was in place, teeth surfaces were cleaned with a polishing brush without paste, rinsed, and dried thoroughly. A container with PRG Barrier Coat BASE was pulled from the package and one drop of PRG Barrier Coat ACTIVE was added to it. The materials were mixed using the disposable tip provided in the kit. Once mixed there were two minutes of working-time as the material became more viscous over time. A thin layer of the mixture was applied to the surface of the tooth and left undisturbed for at least three seconds. The material was light cured for $10 \mathrm{sec}$ according to the manufacture's instruction using 3M ESPE Elipar light curing unit operating at light intensity of 1200 $\mathrm{m} \mathrm{w} / \mathrm{cm}^{2}$ in standard mode. A radiometer was used to verify the output irradiance of the curing unit regularly checked. After light curing, the uncured layer was removed by gently rubbing the surface with a water moistened cotton ball.

\section{Application of sodium fluoride varnish:}

BiFluorid 10 (VOCO) was the used sodium fluoride varnish. The single dose form was used, for optimal standardization of the amount of fluoride varnish used. Concerning the storage of the BiFluorid 10, it was stored in refrigerator at $4{ }^{\circ} \mathrm{C}$ as recommended by the manufacturer. The foil in the single dose unit was pierced using a Micro-Tim brush, the opening was enlarged, and in a circular movement, the brush was being coated with the fluoride varnish. Thin coat was applied on 
the cleaned teeth in the side allocated for sodium fluoride treatment. The varnish left undisturbed from 10-20 seconds then air-dried.

\section{Postoperative evaluation for the dentin hyper- sensitivity}

Teeth were reexamined by the same stimuli as done preoperatively and the data were collected in the participants case report form. The process was repeated at the follow up periods, which were one week and four weeks later. The assessment times were chosen according to (Marto et al. 2019) who conducted a systematic review about the evaluation of the efficacy of dentin hypersensitivity treatment. This review revealed that there was two follow up times either immediate (up to one day) or medium ( 2 to 7 days, 8 to 15 days, or 15 to 30 days). A total number of six participants was dropped out (Five at the first assessment time and one at the second assessment time). The principal investigator filled to contact them after they did not show up.

\section{Statistical analysis}

Statistical analysis was performed using IBM SPSS Statistics Version 2.1 for Windows. Data was presented as mean and standard deviation (SD). The significance level was set at $\mathrm{P} \leq 0.05$. Two-way ANOVA test was performed to evaluate the effect of the two study variables and their interaction on dentin hypersensitivity. The effect size was measured using partial eta-squared $\left(\Pi_{p}^{2}\right.$ to evaluate the strength of the relationship between two groups (Sodium fluoride varnish as a control and PRG barrier coat as intervention . Effect size was interpreted as follows (Field 2013): small (0.01$0.059)$, medium (0.06-0.139) and strong (>0.14). Kruskal-Wallis (non-parametric test, repeated comparisons) followed by Bonferroni-corrected Wilcoxon Signed-Rank test (non-parametric test, 2 related samples) were conducted to compare dentin hypersensitivity assessed by visual analogue scale (VAS) at different assessment time periods within each desensitizing agent. Mann-Whitney test (non- parametric test, 2 independent samples) was used to compare VAS scores between both desensitizing agents at each assessment time.

The absolute risk, relative risk, relative risk reduction with $95 \%$ confidence interval of dentin hypersensitivity were calculated. For each assessment time, the absolute risks of dentin hypersensitivity were compared between the groups using Chi-square test (Hulley et al. in 2013).

\section{Absolute risk (AR) is the probability of chance of an event}

The absolute risk in control group (ARC) equal to the hypersensitivity occur in the sodium fluoride group divided by the total number of teeth received sodium fluoride varnish. The absolute risk in the treatment group (ART) equals the hypersensitivity occur in the giomer varnish group divided by the total number of teeth received giomer varnish.

\section{Absolute risk reduction (ARR) equal to ARC-ART}

\section{Relative risk equals ART / ARC}

\section{Relative risk reduction equal to (ART-ARC)/ARC}

Sodium Fluoride varnish group was used as control group for relative risk calculation.

\section{RESULTS}

Two-way Anova and effect size of desensitizing agent and assessment time on dentin hypersensitivity

Two-way ANOVA results in table (1) showed that the two independent variables "desensitizing agent" and "assessment time" at $\mathrm{P}<0.0001$ and their interaction at 0.006 had a significant effect on dentin hypersensitivity.

The effect size of desensitizing agent and the interaction between "desensitizing agent" and "assessment time" $\left(\Pi_{\mathrm{p}}^{2}=0.085\right.$ and 0.068 respectively) was medium compared to the large effect size of "assessment time" $\left(\Pi_{\mathrm{p}}^{2}=0.459\right)$. 
Effect of desensitizing agent on dentin hypersensitivity at each assessment time:

Table (2) showed mean (SD) of VAS and P-value for the effect of desensitizing agent on dentin hypersensitivity at each assessment time. MannWhitney test showed that at $\mathrm{T}_{1}$ and $\mathrm{T}_{4}$ there was no statistically significant difference between sodium fluoride and giomer both group at $\mathrm{P}=0.465$. As at $\mathrm{T}_{1}$ sodium fluoride revealed mean $(\mathrm{SD})$ equal
$3.39(1.11)$ and giomer revealed mean (SD) equal 3.79(1.51). On the other hand T4, sodium fluoride revealed mean (SD) equal 3.13(1.01) and giomer revealed mean (SD) equal 2.56(1.87) at $\mathrm{P}=0.222$. While at $\mathrm{T}_{2}$ and $\mathrm{T}_{3}$, there was significant difference between two groups. At which Giomer showed lower values $2.17(0.57)$ and $0.17(0.57)$ respectively and the Fluoride revealed higher values 3.21(0.99) and $1.30(0.97)$ respectively.

TABLE (1): Two-way ANOVA and the effect size of desensitizing agent and assessment time on dentin hypersensitivity.

\begin{tabular}{|l|c|c|c|c|c|c|}
\hline \multicolumn{1}{|c|}{ Source } & $\begin{array}{c}\text { Type III Sum } \\
\text { of Squares }\end{array}$ & df & $\begin{array}{c}\text { Mean } \\
\text { Square }\end{array}$ & F & Sig. & $\mathbf{\eta}^{2}$ \\
\hline Corrected model & $249.951^{\mathrm{a}}$ & 7 & 35.707 & 25.547 & $<0.0001^{*}$ & 0.504 \\
\hline Intercept & 1165.049 & 1 & 1165.049 & 833.531 & $<0.0001^{*}$ & 0.826 \\
\hline Desensitizing agent & 22.962 & 1 & 22.962 & 16.428 & $<0.0001^{*}$ & 0.085 \\
\hline Assessment time & 208.973 & 3 & 69.658 & 49.836 & $<0.0001^{*}$ & 0.459 \\
\hline Desensitizing agent x Assessment time & 18.016 & 3 & 6.005 & 4.297 & $0.006^{*}$ & 0.068 \\
\hline Error & 246.000 & 176 & 1.398 & & & \\
\hline Total & 1661.000 & 184 & & & & \\
\hline Corrected Total & 495.951 & 183 & & & & \\
\hline
\end{tabular}

*: significant $(P \leq 0.05)$

TABLE (2): Mean (SD) of VAS and P-value for the effect of desensitizing agent on dentin hypersensitivity at each assessment time using Mann Whitney test.

\begin{tabular}{|l|c|c|c|c|}
\hline & $\mathbf{T}_{1}$ & $\mathbf{T}_{2}$ & $\mathbf{T}_{3}$ & $\mathbf{T}_{4}$ \\
\hline Sodium Fluoride varnish & $3.39(1.11)$ & $3.21(0.99)$ & $1.30(0.97)$ & $3.13(1.01)$ \\
\hline Giomer varnish & $3.79(1.51)$ & $2.17(0.57)$ & $0.17(0.57)$ & $2.56(1.87)$ \\
\hline P-value & $0.465 \mathrm{NS}$ & $<0.0001 *$ & $<0.0001^{*}$ & $0.222 \mathrm{NS}$ \\
\hline
\end{tabular}

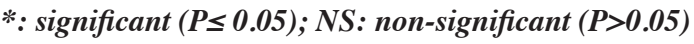




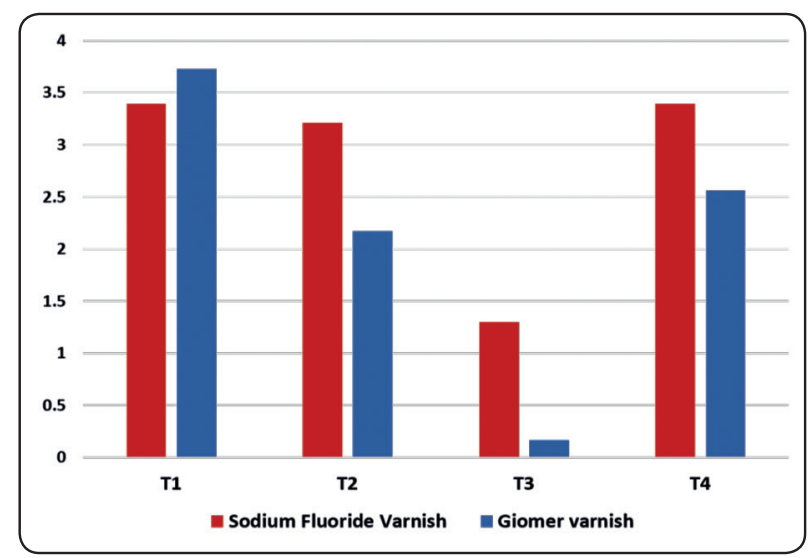

Fig. (1) Bar chart showing VAS scores of both desensitizing agents at each assessment time.

\section{DISCUSSION}

Dentin hypersensitivity resembles a main concern for the dentists and dental patients nowadays. For patients, it is an annoying problem affecting their quality of life while for dentists; it is a problematic issue due to absence of strong evidence regarding the optimal line of treatment. The problem of the pain from exposed dentin to oral environmental factors was termed as dentin hypersensitivity (West et al.in 2014). This condition is developed in two phases: lesion localization and lesion initiation. The scenario is as follow, dentin surface become exposed then the smear layer and smear plug are removed causing the exposure of the dentinal tubules and pulp to different stimuli as chemical, thermal, tactile or osmotic (West et al. in 2013). In the literature, there was many ways for treatment of dentin hypersensitivity by either nerve desensitization, occlusion of the dentinal tubules or by the photobiomodulation action-using laser. Nerve desensitization was performed by using potassium salts that have a direct action on the nerves causing decrease in the excitability of the A fibers present in dentin leading to decrease the dentin hypersensitivity (Vieira and Santiago in 2009).
The occlusion of dentinal tubules can be either physical or chemical. The physical occlusion occurs without performing any form of adhesion within dentinal tubules or the tooth structure like the use of dentin bonding agents or composite resin. While in the chemical occlusion, the used desensitizing agents as fluoride varnish, glutaraldehyde based agents, sodium fluoride iontophoresis and mineralizationpromoting cements form a chemical adhesion for a long period of time than the physical occlusion. According to several studies, the laser considered one of the most recent and effective way to decrease the degree of dentin hypersensitivity. However, it is not widely used as the topically applied agents that are more cost effective, with no need of extra devices and easily applied to the patients (Loveren et al in 2018).

In this study, Giomer varnish (PRG barrier coat) was used as the intervention agent while Sodium fluoride varnish (BiFluorid 10) was the control agent. The sodium fluoride varnish considered as control agent because it is the most popular used desensitizing agent in the treatment of dentin hypersensitivity. The fluoride has many roles in inhibiting the demineralization, enhancing the remineralization and having a strong bactericidal effect. Active free ions of Fluoride when it becomes available in the oral environmental fluids, formation of fluorapatite and calcium fluoride take place. Deposition of fluorapatite crystals dictates the presence of fluoride, calcium and phosphate ions in an adequate amount. While for the formation of calcium fluoride, presence of only calcium and fluoride ions is needed. Furthermore, the concentration of fluoride in the oral fluid and its $\mathrm{pH}$ are other deciding factors for formation of fluorapatite crystals. When it becomes lower than 50-ppm and $\mathrm{pH}$ is more than 4.5, the circumstance becomes favorable to Fluorapatite formation. On the other hand, when the fluoride concentration is more than 100- ppm and $\mathrm{pH}$ is less than 4.5, calcium fluoride is formed as mentioned by Fejerskov et al. in 2008. It is also of great importance to know that, 
the solubility of fluorapatite in the oral fluids is less than calcium fluoride. Solubility of Calcium fluoride is beneficial in reducing the effect of acid attack during low $\mathrm{pH}$ as it release free ionic fluoride ions, which helps in the remineralization, and indirectly in reducing the dentin hypersensitivity.

According to the BiFluorid manufactory instructions, it is consists of $5 \%$ sodium fluoride and $5 \%$ calcium fluoride. The sodium fluoride react with the calcium ion exist in saliva and dentinal tubules causing the formation of calcium fluoride. The collaboration between the two components allow the release of high percent of fluoride due to the presence of $5 \%$ sodium fluoride and long term fluoridation due to the presence of $5 \%$ calcium fluoride. The solubility of $5 \%$ sodium fluoride allow the release of fluoride ions quickly then the fluoride ion transfer to calcium fluoride on the surface of the tooth to permit the remineralization while the relatively low solubility of the $5 \%$ calcium fluoride permit the long lasting effects of the varnish due to its ability of retention on the tooth surface. The BiFluorid 10 stick to the tooth and start to from an immediate protection against any harmful stimuli. Furthermore, it seals the open dentinal tubules by the precipitation of the 5\% calcium fluoride (Petersson in 2013).

Several studies had been conducted to evaluate the desensitizing efficacy of sodium fluoride varnish. Some evaluated its effect per se without any activation or augmentation from external source and others with activation. Aparan et al. in 2010 demonstrated the use of iontophoresis, which is application of small electrical current to the Fluoride agent to stimulate its ionization and release of free active fluoride varnish. Results of this article showed that the combination between iontophoresis and sodium fluoride varnish reduced the dentin hypersensitivity than when it was used alone. Furthermore, Kumar and Mehta in 2005 revealed the significant improvement of Sodium Fluoride varnish when it was augmented with laser application. All these articles reflected the better action of sodium fluoride varnish, when it was used in combination with the external activating source to improve its adhesion and occlusion of dentinal tubules for long period and prevent its easily washing away from the tooth surface by brushing action.

On the other hand, Giomer is considered as a hybrid material between the resins modified glass ionomers and the composite resins. This combination aiming to optimize the properties of both materials therefore, the Giomer has good esthetics with highly finished surface and good mechanical properties together with being one of the bioactive glass materials. It is composed of $45 \% \mathrm{Sio} 2,24.5 \%$ $\mathrm{Na}_{2} \mathrm{O}, 24.5 \% \mathrm{CaO}$ and $6 \% \mathrm{P}_{2} \mathrm{O}_{5}$. The presence of sodium is very important due to its bioactivity while the phosphate is responsible for the degree of degradation and formation of apatite. Based on this composition, giomer has several clinical applications in implant, periodontics, bone generation, pulp capping, Root canal treatment, as restorative materials, in air abrasion, enamel remineralization and finally dentin hypersensitivity as mentioned by Skallevold et al. in 2019. When Giomer varnish become in contact with the oral fluids, it starts to bind to the collagen fiber and release six different types of ions like fluoride, calcium, phosphate that help in the formation of apatite and the release of fluoride to seal the open dentinal tubules (Rusnac et al.2019).

The results of the current study revealed that Giomer is better than the sodium fluoride varnish at $\mathrm{T}_{2}$ (immediately after the application of desensitizing agents) and $\mathrm{T}_{3}$ (one week later) with no significant difference in $\mathrm{T}_{1}$ (Base line) and $\mathrm{T}_{4}$ (one month later). This could be attributed to the bioactive function of the Giomer varnish which is related to the presence of surface pre react glass. Such particles are used as fillers and give the ability of the release of different ions like Fluoride, Sodium, Silica, Aluminum, Strontium and Borate. On release of these ions, Giomer varnish has a remineralizing effect, antibacterial action and acid buffering capacity. In addition, they favor the formation of the fluorapatite crystals which are more acid resistance and less 
soluble than the hydroxyapatite. This result is in agreement with Suzuki et al. in 2015, Samueli et al. in 2017 and Raviskhankar in 2018 who showed the same results with the same explanation and Skallevold et al. in 2019 who conducted that Giomer has resistance against $\mathrm{pH}$ drop and brushing off the occluded dentinal tubules. Due to the resinous nature of Giomer, this allowed it to bond to tooth structure for long period while chemically adhere to the opened dentinal tubules due to the properties taken from glass ionomer as mentioned by Madruga et al. in 2017. No clear evidence about Giomer varnish to disagree with the mentioned explanation due to deficiency in the conduct clinical trials.

It is worth mentioned that, the Sodium Fluoride varnish revealed a desensitizing effect but not exceeding the effect of Giomer varnish due to its chemical action to decrease the movement of fluid inside the dentinal tubules through the precipitation of calcium fluoride crystals, which facilitate the transformation of hydroxyapatite to fluorapatite (Petersson 2013). However, the Sodium fluoride varnish did not have a long lasting effect. It is removed gradually from the affected tooth surface by tooth brushing action or consumption of acidic drinks. This could be related to the solubility of the calcium fluoride that is highly soluble in relation to the fluorapatite crystals and this explanation is in agreement with Torres et al. in 2014.

After one month follow up, it is worth mentioned that Giomer failed to prove its superiority to sodium fluoride varnish statistically according to the calculated P value. Despite clinically, there was an observed difference between the outcome of both desensitizing agents. This point directed us to find alternative statistical methods to confirm either the clinical or the $\mathrm{P}$ value findings. From reviewing the literature, $\mathrm{P}$ value has some drawbacks, as it is not measure the probability that the studied hypothesis is true, or the probability that the data was produced by random chance alone and does not measure the size of an effect or the importance of a result. Therefore, it was much favorable to calculate the absolute risk, relative risk, relative risk reduction and the confidence interval (Hulley et al.2013). Emphasizing these points in the current study revealed $83 \%$ relative risk reduction at $\mathrm{T}_{3}$ and $24 \%$ at $\mathrm{T}_{4}$ for Giomer. It means that the giomer varnish reduced the degree of dentin hypersensitivity by $83 \%$ at $\mathrm{T}_{3}$ and $24 \%$ reduction at $\mathrm{T}_{4}$.

Regarding, the effect of assessment time it was obviously shown that the effect of desensitizing agents was increased after one week of application but decreased by time. In case of Giomer, its desensitizing effect decreased from one week until one month but this decrease did not reach the baseline value as Sodium Fluoride did. This result was with agreement with Papadopoulou et al. in 2019 who demonstrated that Giomer varnish decreased dentin hypersensitivity through sealing the opened dentinal tubules by the released ions that also prevented the growth of streptococcus mutants. However, this effect was detected for a while but relapse occured during post treatment evaluation time (one month and three months). In contrast, Sodium Fluoride showed short lasting effect as mentioned before because most of fluoride was released during the first two weeks then decreased gradually by time. This was in disagreement with Ritter et al. in 2006 who revealed that application of topical fluoride varnish reduced cervical dentin hypersensitivity for 24 weeks. This disagreement might be due to the different formulations of Sodium Fluoride used, which was dissolved in alcoholic solution with resinous nature.

Based on the current findings, null hypothesis of the present study is rejected and it should be mentioned that Giomer varnish is a promising desensitizing agent with a powerful and extended effect. However, shortage in the conducted clinical trials with lack of evidence is the main challenge in providing final clue in relation to Giomer clinical efficiency. So, it is highly recommended to conduct well-formulated clinical trials to investigate the desensitizing effect of Giomer in comparison to different agents with different mode of actions and at different follow up periods. 


\section{CONCLUSIONS}

- Giomer is a powerful desensitizing agent with an intermediate lasting effect.

- Sodium fluoride varnish has a weak shortlasting desensitizing effect.

- Time factor is very important in determining the efficacy of the desensitizing agent.

\section{RECOMMENDATIONS}

- As this clinical trial found that giomer varnish (PRG barrier coat) was more effective than the fluoride in treatment of dentin hypersensitivity. High-quality, well-designed clinical protocols are required to compare the giomer varnish with others different desensitizing agents to reach a definitive recommendations and consensus.

- The use of sodium fluoride as a gold standard for treatment of dentin hypersensitivity showed by reemphasized.

\section{REFERENCES}

- Addy, M. (2002): Dentine hypersensitivity: new perspectives on an old problem. International Dental Journal, 52: 367-375.

- Aparna, S., Setty, S. and Thakur, S. (2010): Comparative efficacy of two treatment modalities for dentinal hypersensitivity: A clinical trial. Indian Journal of Dental Research, 21: 544-548.

- $\quad$ Athuluru, D., Reddy, C., Sudhir, K.M., Kumar, K., Gomasani, S. and Nagarakanti, S. (2017): Evaluation and comparison of efficacy of three desensitizing dentifrices on dentinal hypersensitivity and salivary biochemical characteristics: A randomized controlled trial. Journal of Dental Research, 14: 150-157.

- Bartold, P.M. (2006): Dentinal hypersensitivity. A review. Australian Dental Journal, 51:212- 2188.

- Brännström, M. and Aström, A. (1972): The hydrodynamics of the dentine: Its possible relationship to dentinal pain. International Dental Journal, 22:219-227.

- Chan, A.W., Tetzlaff, J.M., Gotzche, P.C., Atlman, D.G., Mann, H., Berlin, J.A., Dickerin, K., Hrobjartsson, A., Schulz, K.F., Parulekar, W.R., Krleza-Jeric, K., Laupacis,
A. and Moher, D. S. (2013): SPIRIT 2013explanation and elaboration: guidance for protocols of clinical trials. British Medical Journal, 346:1-42.

- Cunha-Cruz, J., Wataha, J. C., Heaton, L.J., Rothen, M., Sobieraj, M., Scott, J. and Berg, J. (2013): The prevalence of dentin hypersensitivity in general dental practices in the northwest United States. Journal of American Dental Association, 144:288-296.

- $\quad$ Fejerskov, O., Kidd, E., Nyvad,B., and Baelum, V. (2008): Dental caries ( The Disease and its Clinical Management, 2 nd edition., p. 210-230, BlackWell Munksgaard.

- Gernhardt, C.R. (2013): How valid and applicable are current diagnostic criteria and assessment methods for dentin hypersensitivity? An overview. Journal of Clinical Oral Investigations, 17:31-40.

- Halabo, S. (2015): Giomer varnish for prolonged hypersensitivity relief. Oral health, 15:1-6

- Hall, C., Mason, S. and Cooke, J. (2017): Exploratory randomised controlled clinical study to evaluate the comparative efficacy of two occluding toothpastes - a 5\% calcium sodium phosphosilicate toothpaste and an $8 \%$ arginine/ calcium carbonate toothpaste - for the longer-term relief of dentine hypersensitivity. Journal of Dentistry, 60: 36-43.

- Hulley, S.B., Cummings, W.S., Browner, W.S., Grady, D.G. and Newman, T.B. (2013): Designing clinical research. $4^{\text {th }}$ edition., p. 151-171 Wolters Kluwer, Lppincott Williams and Wilkins 151-171.

- $\quad$ Kumar, N.G. and Mehta, D.S. (2005): Short-term assessment of the ND: YAG laser with and without sodium fluoride varnish in the treatment of dentin hypersensitivity - A clinical and scanning electron microscopy study. Journal of Periodontol, 76:1140-1147.

- $\quad$ Loveren, C.V., Schmidlin, P.R., Martens, L.C. and Amaechi, B.T. (2018): Dentin hypersensitivity management. Journal of Clinical Dentistry Reviewed, 2:1-10.

- $\quad$ Madruga, M.D., Sliva, A., Rosa. W., Piva, E. and Lumd, R. (2017): Evaluation of dentin hypersensitivity treatment with glass ionomer cements: A randomized clinical trial. Journal of Brazilian Oral Research, 31:1-8.

- Martoa, C.M., Paula, A.B., Nunesa, T., Pimentaa, M., Abrantes, A.M., Pires, A.S., Laranjob, M., Coelhoa, A., Donatof, H., Botelho, M.F., Ferreira, M.M. and Carrilho, E. (2019): Evaluation of the efficacy of dentin hypersensitivity treatments - a systematic review and follow-up analysis. Journal of Oral Rehabilitation, 46:952-990. 
- Orchardson, R. and Collins, W.J.N. (1987): Clinical features of hypersensitive teeth. British Dental Journal, 162: 253-256.

- Orchardson, R. and Gillam, D.G. (2000): The efficacy of potassium salts as agents for treating dentin hypersensitivity. Journal of Orofacial Pain, 14: 9-19.

- $\quad$ Papadopoulou, A., Vourtsa, G., Tolidis, K., Koumpia, E.K., Gerasimou, P., Strakas, D. and Kouros,P. (2019): Clinical evaluation of a fluoride gel, a low-level laser, and a resin varnish at the treatment of dentin hypersensitivity. Lasers in Dental Science, 3:129-135.

- Petersson, L. G. (2013): The role of fluoride in the preventive management of dentin hypersensitivity and root caries. Journal of Clinical Oral Investigations, 17: 63-71.

- $\quad$ Que, K., Ruan, J., Fan, X., Liang, X. and Hu, D. (2010): A multi-centre and cross-sectional study of dentine hypersensitivity in China. Journal of Clinical Periodontology, 37: 631-637.

- $\quad$ Raviskhankar, P., Viswanath, V., Archana, D., Keerthi, V., Dhanapal, S. and Priya, K. (2018): The effect of three desensitizing agents on dentin hypersensitivity: A randomized, split mouth clinical trial. Indian Journal dental research, 29:51-55.

- $\quad$ Ritter, A.V., Dias, W.D., Miguez, P., Caplan, D. and Swift, E.J. (2006): Treating cervical dentin hypersensitivity with fluoride varnish a randomized clinical trial. Journal of American Dental Association, 137:1013-1020.

- Rusnac, M.L., Gaspatik, C., Irimie, A.I., Grecu, A.G., Mesaros, A.S. and Dudea, D. (2019): Giomers in dentistry at the boundary between dental composites and glass ionomers. Journal of Medicine and Pharmacy Reports, 92:123128.
- $\quad$ Samuel, S.R., Khatri, S.G., Acharya, S. and Patil, S.T. (2015): Evaluation of instant desensitization after a single topical application over 30 days: A randomized trial. Journal of Australian Dental Association, 60:336-342.

- Samueli, A., Hill, R.G. and Gillam, D.G. (2017): Bioactive Glasses in the Management of Dentine Hypersensitivity: A Review. Journal of Dental Health Current Research, 3:1-6

- $\quad$ Skallevold, H.E., Rokaya, D., Khurshid, Z. and Zafar, M.S. (2019): Bioactive Glass Application in dentistry. International Journal of Molecular Sciences, 20: 1-24.

- Suzuki, M., Yamada, A., Saito, K., Hino, R., Sugawara, Y., Ono, M., Naruse, M., Arakaki, M. and Fukomoto, S. (2015): Application of a tooth-surface coating material containing pre-reacted glass-ionomer fillers for caries prevention. Pediatric dental journal , $41: 1-7$.

- $\quad$ Torres, C.R., Silva, T.M., Fonseca, B.M., Sales, A.L., Holleben, P., Nicolo, R., and Borges, A.B. (2014): The effect of three desensitizing agents on dentin hypersensitivity: a randomized, split-mouth clinical trial. Journal of Operative Dentistry, 39:186-194.

- Vieria, A.H. and Santiago, S. (2009): Management of dentinal hypersensitivity. Journal of General Dentistry, 66:120-126.

- West, N., Seong, J. and Davies, M. (2014): Dentin hypersensitivity. Monographs in Oral Science,25:108-112.

- West, N.X., Lussi, A., Seong, J. and Hellwing, E. (2013): Dentin hypersensitivity: pain mechanisms and aetiology of exposed cervical dentin. Journal of Clinical Oral Investigation, 17: 9-19.

- Zeolaa, L.F., Soaresa, P.V. and Cruzb, J.C. (2019): Prevalence of dentin hypersensitivity: Systematic review and meta-analysis. Journal of dentistry, 81: 1-6. 